\title{
INNOVACIONES EN LA FORMACIÓN INICIAL DOCENTE Y LOS DESAFÍOS PARA EL DESARROLLO PROFESIONAL DOCENTE
}

\author{
Andrea Figueroa Vargas ${ }^{1}$
}

\section{RESUMEN/ ABSTRACT}

La formación inicial docente se ha visto tensionada a partir de los nuevos requerimientos establecidos en el marco legal chileno. La implementación de la Ley 20.903 (Sistema de Desarrollo Profesional Docente) y la Ley 20.129 (Aseguramiento de la Calidad de la Educación Superior), han permitido avanzar y fortalecer la formación docente de manera sistémica y con ello han surgido nuevos desafíos institucionales, académicos y formativos.

Sobre la base de las implicancias de estas leyes, el artículo da cuenta que la desarticulación entre el saber pedagógico y disciplinar en la formación, continúa siendo una problemática en la formación inicial. En este contexto, los espacios de formación práctica se relevan como instancias reflexivas que aportan tanto a la construcción de los educadores y la integración de dichos saberes, como a elementos identitarios que los distinguen como profesional.

Las necesidades formativas, evidenciadas por los educadores y educadoras en ejercicio profesional, han interpelado a las instituciones formadoras y a la política pública. Esta última, ha respondido con un campo de acompañamiento incipiente en nuestro país: los procesos de inducción y mentoría que, junto a la formación permanente y contextualizada, configuran un nuevo tránsito en la formación de profesores y el desarrollo profesional docente.

Palabras claves: formación inicial docente; desarrollo profesional docente; inserción profesional; inducción; educación superior.

The initial teacher training has been stressed from the new requirements established in the Chilean legal framework. The implementation of Law 20,903 (Teacher Professional

\footnotetext{
${ }^{1}$ Coordinadora Componentes Estándares, Área de Formación Inicial Docente, Centro de Perfeccionamiento, Experimentación e Investigaciones Pedagógicas (CPEIP), Ministerio de Educación de Chile; afigueroav78@hotmail.com.
} 
Development System) and Law 20,129 (Quality Assurance of Higher Education), have made it possible to advance and strengthen teacher training systemically and with it, new institutional challenges have arisen, both academic and training related.

Based on the implications of these laws, the article evaluates that disarticulation between pedagogical and disciplinary knowledge in training continues to be a problem in initial formation. In this context, spaces for practical training are relayed as reflective instances that contribute both to the construction of educators and the integration of said knowledge, as well as to identity elements that distinguish them as professionals.

Training needs, evidenced by educators in professional practice, have challenged the training institutions and public policy. The latter has responded with a field of incipient accompaniment in our country: the processes of induction and mentoring that, together with the permanent and contextualized formation, configure a new transition in teacher training and teacher professional development.

Keywords: Initial teacher training; professional teacher development; professional insertion; induction; higher education.

\section{EDUCACIÓN SUPERIOR, POLÍTICAS PÚBLICAS Y FORMACIÓN INICIAL DOCENTE}

En América Latina, los procesos de masificación de la educación superior han sido progresivos y de creciente complejidad. El siglo XX, en este sentido, ha sido dinámico y han ocurrido cambios de acuerdo a los acontecimientos globales y tendencias educativas en la educación terciaria. En la región, el acceso a la educación superior se ha expresado en tres grandes fases: educación superior de acceso de élite, masificación del sistema y, finalmente, universalización de esta (Rama, 2009; Brunner y Ferrada, 2011).

En la actualidad, existen unas 12.000 instituciones no universitarias de educación terciaria que ofrecen cursos vocacionales, técnicos o tecnológicos (Brunner \& Ferrada, 2011), lo que se expresa en una fuerte diversificación de los sistemas de educación superior, en los cuales existen diferencias en los mecanismos de acceso, el financiamiento, la oferta y el énfasis formativo. Lo anterior ha traído consigo el aumento de la matrícula, la que en los países de Iberoamérica contemplados en el Informe sobre Educación Superior elaborado por CINDA muestra un fuerte dinamismo: entre 1970 y 2008, la población estudiantil se multiplicó más de diez veces, pasando de una matrícula de casi dos millones 
de estudiantes a más de 21 millones. En el mismo período, la población de estudiantes en el mundo había crecido un poco más de cinco veces, de 29 a 159 millones (Brunner \& Ferrada, 2011).

En Chile, la evolución de la matrícula en instituciones de educación superior ha experimentado una dinámica específica de acuerdo al tipo de institución. De la oferta nacional para la educación superior, existen tres tipos de modalidades educacionales para atender al sector productivo: Centros de Formación Técnica, Institutos Profesionales y Universidades. Cada una de estas instancias de formación ha tenido una evolución en su matrícula desde el año 2007 a la fecha con tendencia al aumento en Universidades e Institutos Profesionales. Se evidencia también un leve aumento, entre el año 2009 y 2010 , en la educación en Centros de Formación Técnica cuya matrícula posteriormente ha sido sostenida (Biblioteca del Congreso Nacional, 2011).

Estos antecedentes evidencian las preferencias de los estudiantes respecto de los planes de formación en las diversas instituciones. En este sentido, se observa una clara preferencia de los estudiantes al ingreso a la educación superior, cuya oferta se concentra en carreras profesionales que se ofertan en instituciones públicas estatales y privadas, indistintamente. Lo anterior queda reflejado en el siguiente gráfico:

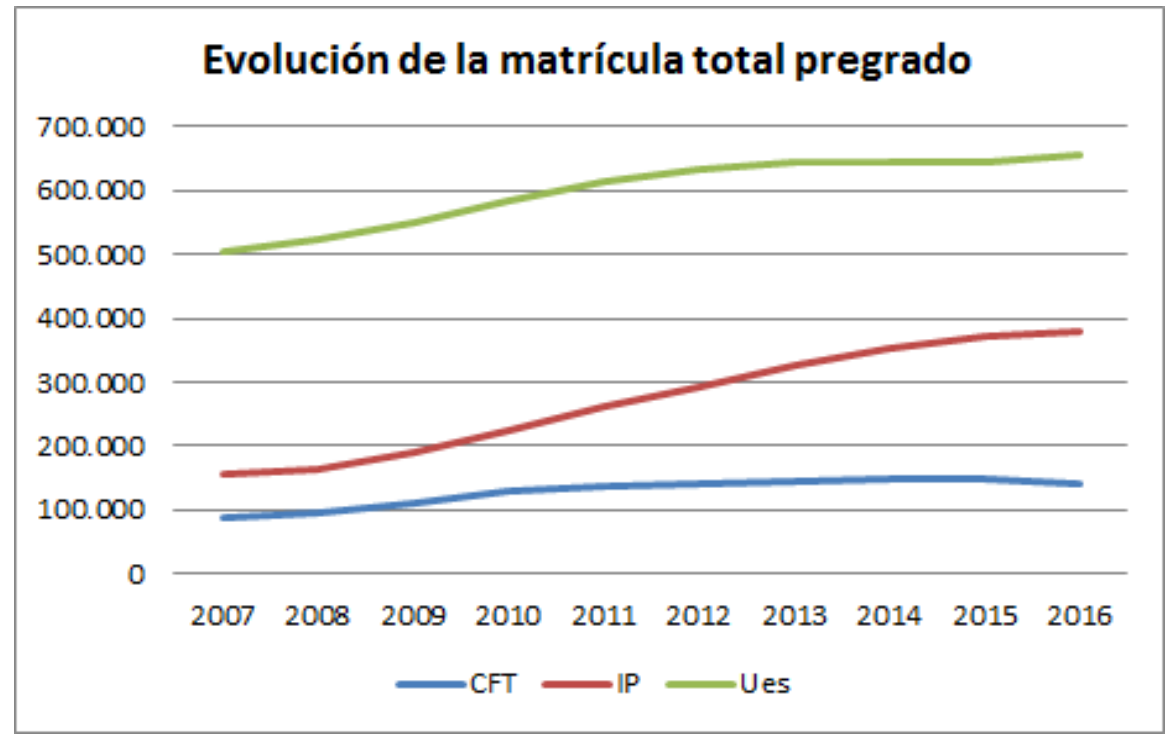

Gráfico 1: Evolución de la matrícula total del sistema de educación superior chileno. Fuente: SIES, 2016. 
En cuanto a la formación inicial docente, la dinámica hasta aquí descrita, en la práctica, ha implicado que un amplio número de ofertas formativas que hoy están adscritas al sistema de educación superior chileno propongan planes de formación con diversos enfoques, énfasis formativos, extensiones y certificaciones. Junto con lo anterior, se ha visualizado en los últimos años cierta tendencia a establecer orientaciones generales que responden y se articulan con aspectos macro de la política pública y que implican innovaciones en la formación inicial docente para responder a la demanda educativa de niños, niñas, jóvenes y personas adultas que viven en una sociedad diversa, multicultural y que se enfrenta a nuevos desafíos.

Las instituciones de educación superior adscritas al Consorcio de Universidades del Estado (CUECH) se han visto impulsadas a innovar sus planes de formación considerando sugerencias y orientaciones que se han considerado de importancia para la formación inicial docente, propuestas principalmente por el Ministerio de Educación. Estas orientaciones, comienzan a generar tensiones en la formación inicial docente, haciendo cada vez más explícito el vínculo de las nuevas políticas con los marcos normativos y los elementos de aseguramiento de la calidad en el macrosistema. Asimismo, se han puesto de relieve temáticas específicas asociadas a demandas sociales en el marco de una sociedad dinámica, pluralista, democrática y que promueve la equidad social, lo que pone de manifiesto el rol de la formación inicial del docente como agente transformador de la cultura y el progreso de la sociedad en su conjunto.

En este marco, el Sistema de Desarrollo Profesional Docente (Ley 20.903) y la Ley de Aseguramiento de la Calidad (Ley 20.129) comienzan a delinear un nuevo camino para la formación inicial. Estas dos leyes, fundamentales en la organización de sistema educativo y la labor de sus docentes, comienzan a trazar los primeros mecanismos para un fortalecimiento sistémico de la formación inicial docente, observando el proceso en la perspectiva de una trayectoria continua que comienza en el momento de ingreso de la/el estudiante en la vida universitaria y que se extenderá durante su ejercicio profesional.

Desde ahí, las casas formadoras han tenido que avanzar sustantivamente, considerando para ello un nuevo escenario: la acreditación obligatoria para las carreras y programas de pedagogía. Ello ha implicado el desarrollo de políticas institucionales, 
procedimientos en el soporte institucional y unidades académicas dinamizadas para redirigir sus esfuerzos al establecimiento de mecanismos sostenidos en el tiempo que garanticen visiones de desarrollo a nivel global.

Otro aspecto relevante asociado a estas transformaciones institucionales concierne a las evaluaciones diagnósticas al inicio y final del proceso formativo de las/os futuros docentes. Estas, de carácter referencial y formativo, implican avanzar en una lógica distinta a la tradicional concepción de la educación superior y desarrollar procesos analíticos a partir de la evidencia, es decir, de la sistematización de resultados objetivos del proceso formativo que permitan diseñar nuevas formas de acompañamiento y nivelación para el avance en el desarrollo de las potencialidades de la/el docente en formación. Desde esta lógica, el espíritu de dichas evaluaciones traza nuevas formas de aproximarse a un desarrollo cada vez más responsable del estudiantado realizando esfuerzos conjuntos en la formación inicial.

Las regulaciones para el ingreso a las carreras y programas de pedagogía surgen como un tercer desafío en la formación de docentes. Ante una gran heterogeneidad de espacios formativos e ingresos no regulados a la educación superior docente, la nueva legislación establece y amplía los mecanismos de ingreso, apuntando a su diversificación: Prueba de Selección Universitaria, ranking, programas de acceso a la educación superior, entre otros.

Finalmente, otro de los elementos que mandata la nueva legislación es la definición de estándares pedagógicos y disciplinarios para la formación inicial docente. Estos estándares, cuya definición, aún en curso, se inició en la administración de la presidenta Michelle Bachelet, apuntan a establecer aquellos conocimientos, habilidades y aptitudes que deben evidenciar las/os egresadas/os de las carreras de pedagogía en los ámbitos pedagógicos y disciplinarios. 


\section{NUDOS CRÍTICOS EN LA FORMACIÓN INICIAL DOCENTE}

Ante los nuevos desafíos y escenarios para la formación inicial docente surge la necesidad y preocupación desde las instituciones de educación superior por responder desde los procesos formativos a las políticas públicas, las necesidades sociales y los requerimientos de los empleadores en el sistema escolar. Esta situación invita a repensar el rol de la universidad en el siglo XXI, como asimismo a replantearse los diseños curriculares tradicionales. Al respecto, Zúñiga, Poblete y Vega (2005) sugieren que

[frente] a estos grandes desafíos, un currículo orientado por competencias puede brindar la oportunidad de abrir un espacio de reflexión sobre procesos de diseño curricular, las prácticas de enseñanza y las formas de evaluación que se llevan a cabo en las entidades de educación superior. (p.30)

En el caso de Chile, sin embargo, luego del tránsito de la educación superior en su conjunto hacia currículums orientados en competencias, vale la pena reflexionar sobre cuál es el impacto que ha tenido la transición a estas construcciones curriculares en la formación inicial docente, y si acaso esta transición efectivamente ha significado transformaciones e innovaciones en los procesos formativos, sus metodologías y procedimientos evaluativos.

Es por ello que la formación de los profesionales para la educación ha sido materia de análisis por parte de las unidades especializadas dependientes del Ministerio de Educación. Dichos análisis se han centrado en el plan de estudios de las carreras y programas de pedagogía, y en las potencialidades de estas instancias para actualizar y promover las innovaciones curriculares necesarias que permitan avanzar a ofertas formativas contextualizadas y articuladas con los territorios escolares, y sintonizadas con las políticas públicas.

Las transformaciones de la formación inicial docente en los últimos años, desde el Programa de Fortalecimiento para la Formación Inicial docente (PFFID) en los años noventa, se han centrado en la necesidad de mejorar la integración entre los aspectos pedagógicos y disciplinarios, y la práctica pedagógica, así como también de visibilizar los elementos comunes que se requiere de un/a docente independientemente del nivel en que 
enseñe. El PFFID ha buscado también estrechar la relación entre las escuelas y las instituciones de educación superior.

Los diagnósticos en esta área hacen referencia a planes de formación desarticulados en lo referido a la formación pedagógica y disciplinar, altamente concentrados en esta última, rígidos, contenidistas, centrados en la enseñanza y no en el aprendizaje entre otros aspectos (Zuñiga, Poblete y Vega, 2009; Dirección de Presupuestos, 2015. Al respecto, González, Herrera y Zurita (2005) plantean que "las universidades han estado tradicionalmente refugiadas en currículos centrados en contenidos y formas de enseñanza obsoletas" (p.16), respondiendo con dificultad a las necesidades de formación de los estudiantes, aspectos evidenciados cada vez con mayor frecuencia en los contextos de aprendizaje en que estos se acercan al ejercicio profesional. Desde esta perspectiva, las temáticas de inclusión y formación ciudadana, y los enfoque de género e intercultural se vuelven relevantes y necesarios para la formación de las/os futuras/os docentes en vistas a su inserción en las nuevas realidades de los territorios escolares. Ante estas necesidades formativas, las instituciones han avanzado con timidez dadas sus estructuras de formación de escaso vínculo con las necesidades del territorio escolar, realidad que ha sido visibilizada y criticada con fuerza desde el mundo estudiantil.

Ante los elementos de contexto que han dinamizado la formación inicial docente, las instituciones de educación superior han desarrollado procesos de rediseño curricular. Estas construcciones, generalmente realizadas de manera colectiva por equipos académicos, comienzan a presentar tensiones en varios aspectos propios de la formación de profesores. Muchas de estas tensiones han sido nudos críticos durante décadas y, en otros casos, tienen que ver con exigencias más recientes que han surgido a propósito de los Planes de Mejoramiento Institucional (PMI) de los establecimientos educativos más que por iniciativas provenientes desde las unidades académicas. Estas tensiones no solo se evidencian en la construcción de planes de formación de las instituciones sin que también en los informes de acreditación de programas y carreras de pedagogía de planes regulares o prosecución de estudios (Comisión Nacional de Acreditación, 2018)..

Señalaremos a continuación algunos de los ámbitos en que se hacen evidentes estas tensiones o nudos críticos. 


\section{PEDAGÓGICO}

En el ámbito pedagógico, base de la formación docente, no tan solo se entregan a los estudiantes herramientas necesarias para construir progresivamente un ethos profesional. Nos encontramos en un momento en el que se aspira a formar docentes que asuman una profesionalidad temprana y se construyan con otras/os como profesionales e intelectuales capaces de reflexionar y analizar críticamente los elementos pedagógicos que influyen en sus prácticas docentes proponiendo nuevas formas para responder al desafío educativo.

Asimismo, la identidad profesional docente en nuestro país (Ávalos y Sotomayor, 2012) es concebida desde los imaginarios de las/os docentes de enseñanza media con un componente vocacional elevado, motivados por su profesión y por enseñar a las/os estudiantes, a pesar de constatar las dificultades que se presentan en esta etapa educativa.

En este marco, no tan solo los aspectos referidos a la política pública y el conocimiento del sistema educativo contribuyen a ello. También el conocimiento sobre aprendizaje y desarrollo de los niños y las niñas, ética profesional, investigación, filosofía, sociología, contextos educativos diversos, currículum y evaluación entregan los elementos fundantes de la profesión; temáticas contempladas en gran parte de los planes de formación del país.

Ahora bien, en el marco de los desafíos del desarrollo profesional docente, estos conocimientos se despliegan y expresan día a día en el ejercicio profesional, en el contexto de la didáctica específica, generando incertidumbres y necesidades de profundización. Ante el nuevo escenario, la ley que crea el Sistema de Desarrollo Profesional Docente (Ley. 20.903) tiene como uno de sus pilares la promoción del desarrollo de los cuerpos docentes mediante la actualización y profundización de sus conocimientos disciplinarios y pedagógicos, la reflexión sobre su práctica profesional, con especial énfasis en la aplicación de técnicas colaborativas con otras/os docentes y profesionales, así como también el desarrollo y fortalecimiento de las competencias para la inclusión educativa (Ley 20.903, art. 11). 
En este plano, las acciones formativas que se ponen a disposición de los docentes de manera gratuita consideran el contexto del territorio escolar con un claro énfasis en desarrollar la reflexión y transformación de las prácticas. Estas acciones formativas buscan, además, integrar elementos clave de la política educacional de nuestro país expresados en la Ley de Inclusión, Género y Formación Ciudadana, teniendo en cuenta también las propias necesidades de los equipos docentes de los establecimientos educacionales.

\section{DIDÁCTICO - DISCIPLINARIO}

El ámbito didáctico - disciplinario ha ocupado, en los últimos años, un lugar cada vez más predominante en los planes de formación. Los rediseños curriculares han tendido a relevar la didáctica haciéndose cargo de las falencias de una formación inicial centrada en conocimientos disciplinarios puros y sin vínculo directo con la enseñanza de los mismos. Se ha avanzado así hacia la enseñanza del contenido de acuerdo a las didácticas propias de las disciplinas, sus resultados de aprendizajes, y sus estrategias y procedimientos evaluativos.

No obstante, en cuanto a nudos críticos sobre la didáctica, existe evidencia robusta sobre el hecho de que la profundidad del conocimiento, aunque escurridiza en su definición y mediciones, parece ser una de las características que afecta la instrucción (Wilson, 1988). En este plano, hay alguna evidencia de que el conocimiento más profundo de la materia resulta en un mayor énfasis en las explicaciones conceptuales (Ball, 1988; Wilson, 1988; Lampert, 1985; Steinberg y otros, 1985).

En este tama, de acuerdo a lo planteado por Grossman, Wilson y Shulman (2005), un número de estudios indica que el conocimiento de la materia per se por parte de las/os docentes experimenta una transformación cuando estas/os comienzan a enseñar, dado que el conocimiento inicial del contenido se enriquece con el conocimiento de las/os estudiantes, del currículum y del contexto de enseñanza (Calderhead y Miller, 1985; Feiman-Nemser y Buchmann, 1985a; Grossman y otros, 1985; McEwan, 1987; Shulman, 1986, 1987; Wilson y otros, 1987), es decir, se ve dinamizado por el contexto escolar y las interacciones de los sujetos en el aula. 
En un sentido complejo del concepto de didáctica, la proyección del campo requiere necesariamente tener presente el espacio escolar, sus características contextuales, los dispositivos de aprendizaje, el estado del arte de la disciplina, los aspectos pedagógicos y la integralidad de estos. Al considerar todos estos elementos se hace evidente la necesidad de avanzar a perfiles académicos diversos a los tradicionales y requeridos por la instancias acreditadoras que apunten y valoren la experiencia en el sistema escolar, y la relevancia de la investigación en acción en contextos escolares orientada a promover nuevas soluciones a los problemas didácticos que surgen en el aula.

Ante las carencias formativas en este ámbito, algunas instituciones han desarrollado programas de posgrado de continuidad que pretenden abordar los nudos críticos en la formación docente, ofreciendo alternativas de educación continua centradas en aspectos didácticos que no fueron contemplados en la formación inicial docente. Estos programas, aunque constituyen una respuesta a las carencias señaladas, no se encuentran aún articulados con las necesidades que evidencia una trayectoria docente.

\section{PRÁCTICA}

La formación práctica a nivel de programas de pedagogía resulta muy disímil. Elementos tales como el peso formativo en el plan de estudios, la estructura, los sentidos, la articulación con las líneas disciplinarias y pedagógicas, las horas y extensión, entre otros, dan cuenta de una línea formativa que ha transitado hacia nuevos sentidos y conceptualizaciones, revitalizando la importancia tradicional de la misma en la formación de docentes. Sin embargo, siguen existiendo nudos críticos cuando se trata de los contenidos más esenciales de la práctica, esto es, el acompañamiento y el vínculo con el sistema educativo.

El sentido final de la formación práctica es el acercamiento progresivo del estudiante a la realidad escolar. Una realidad conocida desde su experiencia escolar pero desconocida en cuanto a asumir un rol profesional temprano, a la aproximación a la realidad educativa desde un punto de vista científico y al rol profesional que se le imprime a sus interacciones con el espacio escolar. Teniendo esto en consideración, los objetivos de 
la práctica deberían ser, por un lado, desarrollar la capacidad reflexiva en los estudiantes y, por otro, ir construyendo progresivamente su identidad profesional.

La reflexión en y sobre la práctica ha marcado los planes de formación en algunas instituciones imprimiendo un sello en la formación de profesores de carácter reflexivo y analítico. Según Molina (2008), se pueden reconocer tres niveles de reflexión: en un primer nivel, se ubica una reflexión de carácter técnico que se dirige solo a las acciones realizadas; un segundo nivel se refiere a una reflexión práctica de carácter didáctico, predominando la planificación; y, por último, existe un nivel de reflexión crítica que considera aspectos de carácter ético, ético político y también de sus efectos en el contexto. En la formación inicial docente, la práctica debería realizar estos tránsitos reflexivos permitiendo el acercamiento a la realidad escolar y a la profesión docente es sus aspectos más técnicos, para desde ahí avanzar a procesos ligados con la enseñanza del contenido y el aprendizaje de los sujetos hasta el posicionamiento de la/el docente como sujeto político que interactúa con otras personas en diversos contextos.

\section{INDUCCIÓN PROFESIONAL}

En este ámbito surge la interrogante ¿cómo lograr afianzar los conocimientos, habilidades y competencias de las/os docentes iniciales que se desenvolverán en contextos complejos y diversos?

La literatura ha entregado evidencias suficientes sobre las dificultades que enfrentan las/os docentes noveles al momento de su inserción en el sistema educacional: el desconocimiento del sistema educativo y sus procesos al interior de los establecimientos, el abordaje de los marcos curriculares y evaluativos, la normativa legal, la integración de disciplina/didáctica y las estrategias para promover aprendizajes en las/os estudiantes (Thompson, 2007; Boerr, 2014; Solís, Núñez. Vásquez, Contreras y Ritterhaussen, 2016).

Una investigación en nuestro país de carácter cualitativo, con 29 docentes principiantes, señaló que los desafíos centrales que ellas/os perciben como nudos críticos en el ejercicio profesional son: dominar el conocimiento pedagógico del contenido, manejar la disciplina, 
considerar las necesidades individuales de las/os estudiantes, evaluar sus trabajos y manejar el currículo y recursos (Flores, 2014).

Considerando estos y otros desafíos que las/os docentes enfrentan en el ejercicio profesional es que, a partir de la promulgación e implementación de la Ley de Desarrollo Profesional Docente, se establecen procesos de inducción para los profesores noveles a cargo de mentores capacitados por el Ministerio de Educación, cuyo objetivo es acompañar y apoyar al docente principiante en su primer año de ejercicio profesional. Se busca, así, el desarrollo de un aprendizaje, una práctica y un sentido de responsabilidad profesional efectivos que facilite su inserción en el desempeño profesional y en la comunidad educativa a la cual se integra (Ley 20.903, art.18G).

Desde este espacio de aprendizaje y resignificación del sentido de ser docente, la alianza entre el/la docente mentor/a, la/el docente principiante y su centro educativo permitirá, entre otras cosas, avanzar en el aprendizaje profesional y en la construcción situada de su saber pedagógico. Es por ello que el equipo directivo del establecimiento educacional, en conjunto con el/la mentor/a, efectuará recomendaciones respecto del desempeño de la/el docente principiante durante el proceso de inducción, las que serán consideradas en las acciones de apoyo formativo que esta/e debe recibir (Ley 20.903, art.18G).

Darling-Hammond y Bransford (2005), citado en Ingvarson (2013), plantean tres preguntas referidas a lo que las/os docentes principiantes necesitan saber y poder hacer, desde la lógica de los conocimientos, habilidades y compromisos profesionales que estas/os evidenciarían en el ejercicio profesional.

¿Qué tipo de conocimiento necesitan tener los profesores eficaces respecto de su materia y del proceso de aprendizaje y desarrollo de sus estudiantes?

¿Qué habilidades necesitan los profesores para procurar experiencias de aprendizaje productivas para un grupo diverso de estudiantes, ofreciendo opiniones informativas acerca de las ideas de los estudiantes y evaluando de modo crítico las propias prácticas docentes para mejorarlas? 
¿Qué compromisos profesionales hacen los profesores para ayudar a cada estudiante a tener éxito y continuar desarrollando sus propios conocimientos y habilidades, como individuos y como miembros de una profesión colectiva? (p.36)

Sin duda, el tránsito de la formación inicial al ejercicio profesional no es una instancia fácil para las/os estudiantes-docentes y tampoco lo es para el centro educativo que los recibe y la institución de educación superior que los forma. Implica una transición fluida entre las instituciones, una articulación directa y corresponsable de las/los docentes principiantes que nutra a la escuela y a la universidad, y una estructura de acompañamiento sostenida que aporte a aproximarse al contexto escolar desde una nueva conceptualización y valorización de la profesión docente en su rol político e intelectual, reconociendo el compromiso personal y social que las/los docentes han expresado históricamente.

\section{A MODO DE CIERRE}

Es relevante que, tanto desde el estado y las políticas públicas, como en las instituciones a cargo de la formación inicial, se profundice en la reflexión y toma de decisiones que permitan potenciar los nudos críticos que acaban de mencionarse. Esto debe realizarse en estrecha conversación con los docentes en formación y en ejercicio, de manera de profundizar la pertinencia y contextualización del desarrollo profesional docente.

Las nuevas leyes y políticas implementadas en el sistema educativo son una importante oportunidad para avanzar en estos desafíos. Sin embargo, deben generarse las condiciones y capacidades que propicien el logro de sus objetivos. 


\section{REFERENCIAS}

Ávalos, B., \& Sotomayor, C. (2012). Cómo ven su identidad los docentes chilenos. Perspectiva Educacional, Formación de Profesores, 51(1), 77- 95.

Biblioteca del Congreso Nacional de Chile (2011). Evolución en el Número de Matrículas del Sistema de Educación Superior, 1983-2010. Extraído de https://www.camara.cl/pdf.aspx?prmTIPO=MANDATOANTECEDENTE\&prmID $=465$

Boerr, I. (2014). El largo camino de convertirse en profesor. Docencia 54, 52-63.

Brunner, J. y Ferrada, R. (2011). Educación Superior en Iberoamérica. Informe 2011. Santiago: Centro Interuniversitario de Desarrollo.

Claderhead, J., \& Miller, E. (1985, September). The integration of subject matter knowledge in student teachers'classroom practice. Paper presented at the annual meeting of the British Educational Research Association.

Comisión Nacional de Acreditación (2018). Carreras de pedagogía: análisis de fortalezas y debilidades en el escenario actual. Serie Estudios sobre Acreditación. Extraído de http://www.investigacion.cnachile.cl/

Dirección de Presupuestos (2015). Informe Final de Evaluación Programa Fomento a la calidad de la Formación Inicial Docente. Extraído de http://www.dipres.gob.cl/597/articles-141238_informe_final.pdf

CINDA (2006). Movilidad estudiantil universitaria. Santiago: Centro Interuniversitario de Desarrollo.

CINDA (2005). Diseño Curricular basado en competencias y aseguramiento de la calidad en Educación Superior. Santiago: Centro Interuniversitario de Desarrollo. 
Grossman y otros (1985). Grossman, P.L., Reynolds, J.A., Ringstaff, C. y Sykes, G. (1985). From English major to English teacher: New approaches to and old problem. Stanford, CA.: Knowledge Growth in Teaching Publications Series, Stanford University, School of Education.

Grossman P, Wilson, S. y Shulman L. (2005). Profesores de Sustancia: El Conocimiento de la Materia para la Enseñanza. Revista currículum y formación del profesorado. 9(2), pp. 1-25.

Feiman-Nemser, S. and Buchmann, M. 1985. Pitfalls of experience in teacher preparation. Teachers College Record, 87(1): 53-65

Flores, C. (2014). Inducción de profesores novatos en Chile: un estudio de caso. Pensamiento Educativo. Revista de Investigación Educacional Latinoamericana, 51 (2), 41-55.

Ingvarson, L. (2013). Estándares de ingreso y certificación inicial docente: la experiencia internacional. Calidad en la Educación 38, 21- 77.

Lampert, M. (1985). How do teachers manage to teach? Perspectives on problems in practice. Harvard Educational Review, 55, 178-194.

Ley 20.903. Crea el sistema de desarrollo profesional docente y modifica otras normas. Diario Oficial de la República de Chile. Santiago, Chile, 1 de abril de 2016.

Ley 20.129. Establece un Sistema Nacional de Aseguramiento de la Calidad de la Educación Superior. Diario Oficial de la República de Chile, Santiago, Chile, 17 de noviembre de 2006.

McEwan, (1987). McEwan, H. (1987). Interpreting the subject domains for students: Towards a rhetorical theory of teaching. Unpublished doctoral dissertation. University of Washington, Seatle.

Molina, P. (2008). Práctica docente progresiva en la formación inicial desde un enfoque reflexivo crítico. En R. Fuentealba y J. Cornejo (Edits.), Prácticas reflexivas para la 
formación profesional docente: ¿qué las hace eficaces? (págs. 13-27). Santiago: Ediciones UCSH.

Rama, C.(2009) La tendencia a la masificación de la cobertura de la educación superior en América Latina. Revista Iberoamericana de Educación, 50, 173- 195.

Solís, M.; Núñez, C.; Vásquez, N.; Contreras, I.; Ritterhaussen, S. (2016). Problemas en la inserción profesional de profesores: necesidad de mentoría. Estudios Pedagógicos 42 (4), 201- 221.

Shulman, L.S. (1986). Those who understand: knowledge growth in teaching, Educational Researcher, 15 (2), 4-14. Trad. y edición española ("El saber y entender de la profesión docente") Estudios Públicos 99, 195-224.

Shulman, L.S. (1987). Knowledge and teaching: foundations of the new reforms, Harvard Educational Review, 57(1), 1-22. Edición en español: (“Conocimiento y enseñanza: fundamentos de la nueva reforma"), Profesorado. Revista de Currículum y Formación del Profesorado, 9 (2), 1- 22.

Steinberg, R., Marks, R., y Haymore, J. (1985). Teachers' knowledge and structuring of content in mathematics. Technical Report of Knowledge Growth in a Profession Research Project. Stanford, CA.: School of Education, Stanford University.

Thompson, J. (2007). The first year teacher's survival guide. San Francisco: Jossey - Bass.

Wilson, 1988 Wilson, S.M. (1988). Representations of knowledge in teaching: A case of American history. Unpublished doctoral dissertation, Stanford University, Stanford, CA.

Wilson, S.M., Shulman, L.S. y Richert, A.E. (1987). '150 different ways' of knowing: Representations of knowledge in teaching. En J. Calderhead (Ed.), Exploring Teacher Thinking. London: Cassell, 104-124.

Zúñiga, M. Poblete, A. y Vega. A. (2009). El desarrollo de competencias en un contexto de aseguramiento de Calidad. Extraído de 
http://www.academia.edu/5022466/EL_DESARROLLO_DE_COMPETENCIAS_E N_UN_CONTEXTO_DE_ASEGURAMIENTO_DE_CALIDAD 